\title{
EI CONFLICTO ARMADO EN COLOMBIA Y SUS REALIDADES INVISIBLES
}

\author{
Natalie Rozo Gutiérrez ${ }^{1}$, Ana Cecilia Becerra Pabón ${ }^{2}$, Maritza Barroso Niño ${ }^{3}$ María Antonina Román Ochoa ${ }^{4}$, \\ Esperanza Durán de Camperos ${ }^{5}$, Mancel Enrique Martínez Durán ${ }^{6}$
}

\section{RESUMEN}

En el conflicto armado existen factores sociales y culturales invisibles para la sociedad, lo cual hace imposible la creación de programas de atención social y en salud que den cuenta de las realidades que viven las personas. Es decir, una cosa es lo que piensa el gobierno de turno que necesitan las poblaciones víctimas de la violencia en Colombia y otra cosa muy diferente son las realidades que viven las personas en sus comunidades. El objetivo de este documento es demostrar cómo los aspectos sociales y culturales de una población víctima de la violencia en Colombia son determinantes para el desarrollo social y el éxito de los procesos de reparación. Resultados: Se identificó una categoría núcleo: "Realidades invisibles del conflicto armado en Colombia"; y cuatro categorías emergentes; "Poder social", "Estado y soberanía", Salud mental, individual y familiar" y "Productividad territorial". Conclusiones: La búsqueda de realidades invisibles del conflicto armado en Colombia, nos permite encontrar personas subsumidas en la cultura de la destrucción, con daños en su alma, suplicando mínimas condiciones para vivir, sin palabras para expresar su dolor, sin procesos solidarios y comunales, sin estructuras para el progreso; quienes en su precaria dignidad humana se someten ahora a una reparación que promete igual tratamiento al territorio, la persona, la familia y los colectivos.

Este artículo da cuenta de la fase cualitativa de la investigación denominada: Programa de atención psicosocial y salud integral a víctimas del conflicto armado, Algeciras (Huila), Colombia 2018.

Palabras Clave: Violencia, Análisis cualitativo, Fenomenología.

\section{THE WARFARE IN COLOMBIA AND ITS INVISIBLE REALITIES.}

\begin{abstract}
In the warfare, there are social and cultural factors invisible for society in general. Because of that, it is difficult to create social and health care programs that meet requirements from people who face directly the warfare in small and far away communities. For that reason, the governments focus their attention in what they think it is what people from these communities need instead of being aware and work for what these communities actually needs. The purpose of this document is to demonstrate how the social and cultural aspects of a population victim of violence in Colombia are determinants for social development and the success of reparation processes. Results: A core category was identified: "Invisible realities of the armed conflict in Colombia"; and four emerging categories; "Social power," "State and sovereignty," Mental, individual and family health "and" Territorial productivity. "Conclusions: The search for invisible realities of the warfare in Colombia, allows us to find people immerse in a culture of destruction, with damage in their souls, getting used to minimal conditions to live, without words to express their pain, without solidarity and communal processes, without structures for progress; who in their precarious human dignity now undergo a repair that promises equal treatment to the territory, the person, family and groups.

\footnotetext{
1 Natalie Rozo Gutiérrez, Enfermera, Magíster en salud pública. Docente Escuela de Enfermería. Fundación Universitaria Juan N Corpas.

2 Ana Cecilia Becerra Pabón, Enfermera, Doctora en educación. Coordinadora de Investigación. Escuela de Enfermería Fundación Universitaria Juan N Corpas.

${ }^{3}$ Maritza Barroso Niño. Enfermera, Especialista en salud mental. Docente Escuela de Enfermería. Fundación Universitaria Juan N Corpas.

${ }^{4}$ María Antonina Román Ochoa. Enfermera, Doctora en educación. Decana. Escuela de Enfermería Fundación Universitaria Juan N Corpas.

${ }^{5}$ Esperanza Durán de Camperos. Enfermera, Doctoranda en educación. Docente. Escuela de Enfermería Fundación Universitaria Juan N Corpas.

${ }^{6}$ Mancel Enrique Martínez Durán. Médico, Doctor en ciencias sociales y humanas. Docente. Escuela de Medicina. Fundación Universitaria Juan N Corpas.
} 
This article gives an account of the qualitative phase of the selected research: Program of psychosocial and integral health care for victims of the armed conflict, Algeciras (Huila), Colombia 2018.

Key Words: Violence, Qualitative analysis, Phenomenology.

\section{Recibido 03-02-2019. Aceptado 06- 04-2019 DOI: https://doi.org/10.26752/revistaparadigmassh.v1i1.450}

\section{Introducción:}

La Unidad para la atención y reparación integral a las víctimas afirma que hasta el 2014 el conflicto armado que ha vivido Colombia ha dejado más de siete millones de víctimas, ocasionando un impacto en cerca del 15\% de la población(Moreno Camacho \& Díaz Rico, 2016). Huila es considerado uno de los departamentos con mayor afectación por el conflicto armado en Colombia y, en este Departamento, los municipios con más altos índices de registro de víctimas son Neiva, con 119 (Mayor receptor) y Algeciras con 62 ..." "Neiva y Algeciras los Municipios Con Mayor Victimas del Conflicto Armado en el Huila," n.d.) El conflicto armado interno ha generado baja productividad económica, desintegración social, despojo, daños materiales, emocionales y psicológicos, morales, políticos, socioculturales y en salud de la población (Ramirez et al., 2016), manifestados, entre otros, por ideación suicida, ataques de pánico, desórdenes alimenticios, incremento en el consumo de bebidas alcohólicas y sustancias psicoactivas (López López, Pérez Durán, \& Pineda Marin, 2016).

Es necesario desarrollar mecanismos que contribuyan a la reparación, atención psicosocial y alivio del sufrimiento de los afectados por el conflicto. El Ministerio de Salud y Protección Social de Colombia definió en el 2012 el Programa de Atención Psicosocial y Salud Integral a Víctimas, el cual establece las directrices, estrategias y acciones nacionales para la operatividad y ejecución del programa con el propósito de garantizar "El restablecimiento de las condiciones físicas y psicosociales de la población víctima y la garantía del derecho a la atención en salud física, mental y psicosocial... "("Programa de atención psicosocial y salud integral a víctimas - PAPSIVI," n.d.).

La necesidad de que los municipios con población víctima del conflicto armado, como Algeciras (Huila), desarrollen y ejecuten los lineamientos nacionales de acuerdo con sus características sociales, económicas, demográficas, culturales y políticas constituyó la principal motivación para la realización de esta investigación cuyo objetivo es el de elaborar un Programa de atención psicosocial y salud integral a víctimas del conflicto armado residentesdelacabeceramunicipaldeAlgeciras (Huila), Colombia para la reconstrucción del tejido social de la población con articulación intersectorial e interinstitucional.

\section{Participantes y método}

- Diseño: Estudio cualitativo que se llevó a cabo entre febrero y marzo de 2017 a través de entrevistas semiestructuradas a 8 grupos focales. La duración de cada entrevista estuvo entre 30 y 50 minutos. Las entrevistas a los grupos focales se llevaron a cabo en la población de Algeciras (Huila)-Colombia. Teniendo en cuenta las características de la población (víctimas de violencia) y el contexto 
social en el que se desarrolla la investigación, se hace una variación de la guía de preguntas y de la entrevista, de la siguiente forma:

1. Se establecen unos "estímulos sensoriales" (dibujos), basados en la fase cuantitativa de la investigación: Seguridad, Educación, Alimentación, Salud (sintomatología psicosocial y acceso a los servicios), Ingresos, Reunificación familiar, Vivienda, Rehabilitación, y Participación comunitaria. Que para esta fase se convierten en las pre categorías.

2. Los participantes se involucran en dinámicas lúdicas, en donde debe priorizar sus necesidades de salud en una pirámide colocada sobre una pared. (foto)

3. Una vez todos los participantes hubieran hecho el ejercicio de priorizar sus necesidades, se promueve el análisis y/o reflexión en torno al resultado final de la pirámide.

4. Al finalizar el ejercicio cada participante debe escribir a mano en una hoja de papel su propuesta de mejoramiento de la situación encontrada.

\section{- Muestra y participantes y/o contextos:}

La investigación se llevó a cabo en la población de Algeciras (Huila)-Colombia. Huila, es considerado uno de los departamentos del país con mayor afectación de personas por el conflicto armado en Colombia, su explicación estaba basada "en las diversas causas estructurales y coyunturales de carácter socioeconómico, político y de seguridad, aunque su ubicación geoestratégica es también una de las razones de la presencia de diversos grupos armados ilegales y del conflicto armado, lo cual ha tenido un impacto directo en la gobernabilidad del departamento y en su población"(Bruno et al., 2010).

Para esta fase de la investigación, se realizó muestreo intencional teniendo en cuenta las personas que en la fase cuantitativa de la investigación se catalogaran como "población víctima de la violencia". Los participantes aceptaron la participación voluntaria en la entrevista por medio de la firma de un consentimiento informado. La entrevista fue dirigida por una entrevistadora cualificada, de profesión enfermera y con amplia experiencia en salud mental. Apoyaron el proceso estudiante de Enfermería del semillero de investigación SIEC (Semillero de investigación Estudiantes para el Cuidado) pertenecientes al grupo CUIDARTE.

Se realizaron entrevistas a ocho grupos focales las cuales fueron suficientes para obtener la saturación de la información.

- Análisis: La información se analizó desde la perspectiva metodológica de Giorgi (Giorgi, Giorgi, \& Morley, 2017). De acuerdo con esta perspectiva se tuvieron en cuenta cinco pasos:

1. Lectura de la transcripción con el fin de captar el sentido básico de lo descrito.

2. Reducción fenomenológica científica.

3. Determinación de las unidades de significado.

4. Transformación de las unidades de significado en expresiones que resaltan los significados psicológicos vivido por los participantes.

5. Transformación del significado de las 
expresiones con base en la psicología para describir la estructura de la experiencia.

Surgieron cuatro unidades de significado: Poder Social, Estado y soberanía, Salud mental, individual y familiar, Productividad territorial. Aspectos de rigor como la auditabilidad se tuvieron en cuenta en esta investigación al hacer las grabaciones de toda la entrevista, las transcripciones inmediatas de las entrevistas y describir las características de los informantes y su proceso de selección para participar en los grupos focales.

\section{- Aspectos éticos}

De acuerdo con la resolución 008430 de 1993 (Colombia), esta investigación se clasificó con riesgo mínimo porque emplea el registro de datos a través de procedimientos comunes consistentes con diagnóstico (De Salud, 1993). Se utilizó consentimiento informado, de acuerdo al aval del comité de ética institucional de la Fundación Universitaria Juan N. Corpas.

La investigación se desarrolló con seres humanos, reconociendo el respeto a la vida, a la libertad y a la igualdad como valores que anteceden la dignidad y los derechos humanos, protegiendo así el bienestar de los individuos, aplicando los criterios de beneficencia, no maleficencia, justicia y autonomía (Ministerio de Educación, 2004). Los sujetos de investigación fueron personas mayores de 18 años, quienes dieron su consentimiento para participar en el estudio ("Declaración de Helsinki de la AMM - Principios éticos para las investigaciones médicas en seres humanos WMA - The World Medical Association," n.d.). La investigación fue financiada por el proyecto 1000 K y la Fundación Universitaria Juan N. Corpas.

\section{Resultados y discusión}

En todos los relatos que hicieran los grupos focales, fue evidente la distancia entre la realidad de las víctimas y la versión oficial de los episodios de violencia. Los medios de comunicación pasan por alto la vivencia y afectación de las personas, su repercusión en la cotidianidad de las familias y las comunidades. Así se revela esta categoría núcleo "Realidades invisibles del conflicto armado en Colombia".

Con este sustrato en común, realidades de similar afectación nos van revelando cuatro unidades de significado, que exponemos a continuación:

\section{- Estructuras de poder social}

Tres ingredientes dinamizan el conflicto armado en Colombia: la inequidad, el narcotráfico y el régimen semi-represivo. Quienes ostentan el poder, dice Foucault, lo ejercen como una estrategia. Entonces tiene sentido, en primera instancia, la muerte. Las personas narran cómo se dispone "estratégicamente" de la vida, ya que subyace la idea de que el poder tiene como misión fundamental la defensa de la sociedad. (Salas Salazar, 2014) " No volvieron!... la razón es que ellos están muertos, que les dieron en un combate". (G:5, R23). Es un poder sin fronteras que trasciende culturas, generaciones, ideales, intereses y su esencia se mantiene en el tiempo.

"Empiezo a decir que hemos vivido una guerra en todo el país, icierto! en mi caso llevo más de 30 años, más de 50 años sufriendo la inclemencia de la guerra, hemos perdido" (G:01, R15). De otro lado, Las víctimas del conflicto perciben que ante la pobreza (causa y efecto del conflicto 
armado en Colombia) no existe salida inmediata y que cada día deben comer y dar de comer a sus hijos ("Conflicto armado en Colombia. Conoce sus consecuencias sociales | InspirAction," n.d.).

"iPerdimos todo!" (G:02, R32) "Uno tiene las cosas, vienen las FARC a quitarnos lo que tenemos, ¡es muy duro!" (G2, P3, R4) El poder no solo dispone de la vida y los recursos individuales y familiares, también se apropia de la forma de vida, su calidad y sentido. Se ejerce un control político, que ordena territorios $y$ poblaciones. Normas para convivir bajo control: amenazado, vulnerable y protegido, todos los estados son indeseables. No existe la vida comunitaria ni social. La vida debe desarrollarse solamente en privado.

"... aquí se enseña a vivir y vive porque uno se volvió "Shakira" [...] Sordo, ciego y mudo. Sí así es, mire y calle" (G:02, R3). "[...], usted no oye, no ve, no sabe" (G:02, R27). Las formas de vida social tan determinadas quedan condicionadas. Por tanto, la muerte, y los rituales asociados a la misma (el duelo, la búsqueda de los restos mortales, el velorio, y los demás actos mortuorios), se convierten en el único factor en común, entre quienes han perdido a sus seres amados en el conflicto armado y político, estas son las mujeres y niños viudas de los actores armados (Rojas Tovar, 2006)

\section{- Estado y soberanía}

Los habitantes de Algeciras (Huila) reconocen la ley de: "ellos...los de arriba" [interpretación de los autores], porque la guerrilla se ubica en las montañas y emiten comunicados regularmente. El respetoalavidaestámediado por el cumplimiento de órdenes. Cuando se dio por terminado el conflicto armado, no hizo presencia el estado; "este pueblo ha sido un pueblo olvidado por el gobierno, aquí hemos sido totalmente olvidados (G:01, R18).

No se encuentra una relación de los individuos con el estado como ciudadanos sujetos de derechos, tampoco se aprecian ejercicios ciudadanos o formas de organización que permitan participación en las decisiones municipales o departamentales, no existió una sola vez la palabra democracia ni se encontró en las conversaciones la idea de gobernanza o relaciones políticas con el estado. Así la exigibilidad de los derechos es inconcebible. Identifica sus derechos como necesidades y suplica favores, mendiga o pelea por beneficios, busca quedar en las listas de víctimas, a ver si se logra algo para salir de esta forma de vida. Al respecto se mencionó: "Hay esperanza porque habiendo paz y uno hablando con las autoridades y con todas estas modificaciones entonces ya uno tiene la esperanza que hay alguien que se conduela de uno y espera una ayuda" (G:5, R29).

Y es que si se tiene en cuenta la Declaración Universal de Derechos Humanos (ACNUDH, 2016), es innegable que el estado no ha logrado atender con dignidad los derechos de los pobladores de la región. Como, por ejemplo:

"La salud: es tanto el desorden. ¿No se podrá hacerlo con más orden? Es que aquí, si ni hablar" (G: 01, P: 02 R; 03).

"... que tengamos la vivienda, sin vivienda no hay familia, a la familia le agregamos un poquito de educación y ahí si ..." (G:01, R27).

\section{- Salud Mental individual, familiar y comunitaria.}

Esta categoría emerge mostrando que la vida social y/o comunitaria, es más que la reunión 
de personas, sin importar su permanencia o transitoriedad, y que, a su vez, entraña desarrollos mentales del individuo, afectivos de la familia y lazos en la comunidad.

La OMS cuando define violencia menciona como sus consecuencias el daño psicológico y los problemas de desarrollo (Organization \& Switzerland, 2002); Algeciras (Huila) tiene una guerra de 50 años, es decir una gran parte de su población tiene toda su vida subsumida en el conflicto armado. Estos daños que ha producido la violencia, la pobreza, la marginación, la falta de educación y trabajo los vimos en los grupos focales, cuya afectación en el área cognitiva fue notoria.

"El pueblo está muy desunido". (G:01, R09); "Lo principal tener una comunidad unida", (G: 01, R32).

Aquí, vale la pena tomar en cuenta las reflexiones de Peláez Jaramillo (Peláez Jaramillo, 2011), reconociendo que el psicoanálisis aporta a la comprensión del sujeto, cuando "define la personalidad en términos de estructura y no de atributos", porque los fenómenos descritos por los entrevistados no se pueden comprender como descripción de emociones, reflexiones, percepciones de una realidad, sino que tienen que situar este fenómeno como una afectación tanto en la estructura social como en la estructura misma de los individuos.

Este momento que el país ha llamado postconflicto, llena de esperanzas a las personas y los colectivos. A los grupos focales asistieron dos líderes comunales que mostraban gran interés en gestionar desde la comunidad este momento de reparación.

"Estas reuniones por lo general no se hacian" (G:01, R02). "Y estamos, más sin embargo luchando y estamos todavía volteando como líder, ayudando, bregando a colaborar con la comunidad, con las comunidades", (G:01, R17)

Si es posible reunirse, entonces es posible la escucha. Hablar entraña un propósito terapéutico, pensarse, exponerse, puesto que la palabra tiene el empuje al mundo interior, nos permite simbolizar "Y, lo más importante, permitirles hablar de lo que vivieron y facilitar la reflexión en la comunidad, combatiendo la estigmatización y promoviendo actitudes positivas que permitan el reconocimiento de las víctimas" OMS (2004) citado en (Sentencia T-045, 2010) ("Programa de atención psicosocial y salud integral a víctimas - PAPSIVI," n.d.). "Refresca la vida de uno, al devolver el casete y recordar" (G: 01, R39).

Otro elemento significativo en la salud mental de los habitantes de Algeciras (Huila), tiene que ver con el efecto en la composición familiar, por muerte o desplazamiento. Puesto que cuando las personas pierden integrantes de la familia, calidad del funcionamiento o franca desintegración de la familia, se debe suponer una afectación en su Salud Mental. Al respecto se encontraron los siguientes relatos:

"No tengo familia, los dos hermanos que me ayudaban se los llevó la guerrilla, los cogieron ellos eran trabajadores comunes, itrabajadores! hicieron una recogida y se los llevaron a juntos". (G:5, R22), es una declaración que se acompaña de llanto y que expresa resignación y desesperanza. "Uno no sabe en qué momento le dicen tienen que irse y se tiene que ir". (G:02, R31).

"¿desde pequeña tocarme la violencia como me tocó? lo marca de por vida y pues no sé a toda mi familia le afectó mucho, y yo creo que aparte de la guerra que está viviendo acá... pues digo que eso 
sí me marcó, me marcó mucho al ver a mi familia como sufrió (G:02, R16).

\section{- Productividad territorial}

En todos los grupos focales pronunciaron con orgullo que Algeciras era la despensa del país, la calidad de sus productos agrícolas, la variedad y diversidad también en ganadería, hacían de su producción en carnes y lácteos una región bien reconocida. Al respecto se mencionó: "Este es un pueblo agrícola más que todo, no es ganadero, no es cocalero" (G:02, R11)

Las personas también se refirieron a su geografíayriquezanatural.Sereconocencomo una región con vocación agrícola. Quisieran lograr buenas cosechas y sobretodo formas de comercializar, vías y medios de transporte que permitan llegar a los grandes mercados de ciudades capitales. Definitivamente reconocen en el emprendimiento un camino de libertad y calidad de vida para todas las personas, además, lo expresan como el progreso regional.

\section{Conclusiones, utilidad y limitaciones:}

Aspectos sociales trascendentales condicionan las conductas de los pobladores. Fenómenos como la disputa de territorios y el abandono del mismo por parte del estado legalmente constituido hacen que se genere un "para estado", en donde son los grupos insurgentes los que tienen el poder, emiten normas, toman el control territorial y mediante amenazas y muerte ejercen el poder y generan miedo en la población. El miedo a la muerte propia y de familiares y seres queridos hacen que se generen fenómenos como el desplazamiento, el aislamiento y confinamiento al espacio privado, el silencio y la obediencia. Estas conductas limitan acciones de emprendimiento y superación de la vulneración social y condena a sus habitantes a padecer un eterno sentimiento de victimización y de imposibilidad de acción.

\section{Puntos clave}

Lo conocido sobre tema: Son varias las perspectivas investigativas con enfoque cuantitativo que se ha hecho del fenómeno de la violencia en Colombia y con base en estos resultados es que se han llevado a cabo los planes de mejoramiento y reparación de víctimas que adelanta el gobierno en la actualidad. Pero son pocos los estudios que abordan la problemática desde una perspectiva cualitativa, que profundice en las vivencias de los pobladores y que deje ver el fenómeno desde la profundidad de la emocionalidad.

Qué aporta este estudio: La población de Algeciras (Huila) ha sido fuertemente afectada por las dinámicas sociales de violencia que ha vivido y que vive en la actualidad. Es necesario tenerencuentalavozdelas personas contando sus vivencias, sus miedos y sus esperanzas, para de esta manera crear programas de reparación acordes y pertinentes con las realidades sociales de sus habitantes, y que en realidad logren un impacto en sus condiciones de salud y de vida.

\section{Referencias Bibliográficas}

1. ACNUDH. (2016). Declaración Universal de los Derechos Humanos. Retrieved January 2, 2019, from Oficina del alto comisionado -Naciones Unidas website: https://www.ohchr.org/SP/UDHR/Pages/ UDHRIndex.aspx

2. Bruno, S., Moro, B., Del Carmen, M., Directora, S., Elaborado Por Área De Paz, P. A. I., Alessandro, R., Enriquez, P. (2010). Huila: Análisis de la conflictividad. Retrieved from www.impresolediciones.com

3. Conflicto armado en Colombia. Conoce sus 
consecuencias sociales | InspirAction. (n.d.). Retrieved October 18, 2019, from https://www. inspiraction.org/justicia-economica/conflictoarmado-en-colombia

4. De Salud, M. Resolución Numero 8430 De 1993. (1993).

5. Declaración de Helsinki de la AMM - Principios éticos para las investigaciones médicas en seres humanos - WMA - The World Medical Association. (n.d.). Retrieved October 18, 2019, from https:// www.wma.net/es/policies-post/declaracion-dehelsinki-de-la-amm-principios-eticos-para-lasinvestigaciones-medicas-en-seres-humanos/

6. Giorgi, A., Giorgi, B., \& Morley, J. (2017). The Descriptive Phenomenological Psychological Method In: The SAGE Handbook of Qualitative Research in Psychology (2 Ed.; Willig and Rogers, Ed.). https://doi. org/10.4135/9781526405555

7. López López, W., Pérez Durán, C., \& Pineda Marin, C. (2016). Relación entre el perdón, la reconciliación y la salud mental de las víctimas de la violencia socio-política. Revista de Victimología, (3), 141159. Retrieved from http://www.huygens.es/ journals/index.php/revista-de-victimologia/article/ view/39/23

8. Ministerio de Educación, C. (2004). Ley 911 de 2014. Retrieved from https://www.mineducacion.gov. co/1759/articles-105034_archivo_pdf.pdf

9. Moreno Camacho, M. A., \& Díaz Rico, M. E. (2016). Posturas en la atención psicosocial a víctimas del conflicto armado en Colombia. Agora U.S.B., 16(1), 193. https://doi.org/10.21500/16578031.2172

10. NeivayAlgeciras los Municipios Con MayorVictimas del Conflicto Armado en el Huila. (n.d.). Retrieved May 15, 2017, from http://www.personerianeiva. gov.co/index.php/neiva-y-algeciras-los-municipioscon-mayor-victimas-del-conflicto-armado-en-elhuila

11. Organization, W. H., \& Switzerland. (2002). World report on violence and health. Retrieved from https://www.ncjrs.gov/App/Publications/abstract. aspx?!D=264644

12. Peláez Jaramillo, G. P. (2011). El sujeto y el lazo social en el psicoanálisis | Peláez Jaramillo | Affectio Societatis. Revista Affectio Societatis, 8(15), Art. 19. Retrieved from http://aprendeenlinea.udea.edu. co/revistas/index.php/affectiosocietatis/article/ view/10796/9970

13. Programa de atención psicosocial y salud integral a víctimas - PAPSIVI. (n.d.). Retrieved July 20, 2018, from https://www.minsalud.gov.co/ proteccionsocial/Paginas/Victimas_PAPSIVI.aspx
14. Ramirez, N. H., Juárez, F., Baños, A. J. P., Luzardo, J. G., Chávez, Y. M. R., Castilla, A. M. S., \& Amaya, M. V. V. (2016). Afectaciones psicológicas, estrategias de afrontamiento y niveles de resiliencia de adultos expuestos al conflicto armado en Colombia. Revista Colombiana de Psicologia, 25(1), 125-140. https:// doi.org/10.15446/rcp.v25n1.49966

15. Rojas Tovar, P. (2006). Las viudas del conflicto armado en Colombia: memorias y relatos (I. C. de A. e H. : COLCIENCIAS, Ed.). Bogotá.

16. Salas Salazar, L. G. (2014). Lógicas territoriales y relaciones de poder en el espacio de los actores armados: un aporte desde la geografía política al estudio de la violencia y el conflicto armado en Colombia, 1990-2012. Cuadernos de Geografía: Revista Colombiana de Geografía, 24(1), 157-172. https://doi.org/10.15446/rcdg.v24n1.47777 